names. The Biomek FX liquid-handling workstation from Beckman Coulter, used extensively in DNA preparation, is a sophisticated descendant of the Biomek 1000 system introduced in 1986 . The latest model can simultaneously automate three liquid handlers on the same unit.

The popular BioRobot series of automated workstations made by QIAGEN Instruments near Zurich, Switzerland, offers a range of dedicated platforms for highthroughput genomics. The most recent entrant, the BioRobot Protein LS system, integrates all the software and hardware needed to purify and prepare proteins for study using X-ray crystallography or nuclear magnetic resonance.

Zymark, based in Hopkinton, Massachusetts, continues to develop its Staccato range of liquid-handling workstations, based around the Sciclone liquid-handling robots. The Sciclone ALH3000, launched in autumn last year, can dispense into microplates of up to 1,536 wells, with a new head design that can be rapidly switched to pipette volumes of 1 millilitre down to 100 nanolitres.

"The general trend we are seeing is to integrate a greater number of functions into a single system that can carry out a related set of applications," says Mark Roskey, vice-president of marketing at Zymark.

Advances in detection technology are also boosting the capabilities of workstations. The plate::screen workstation

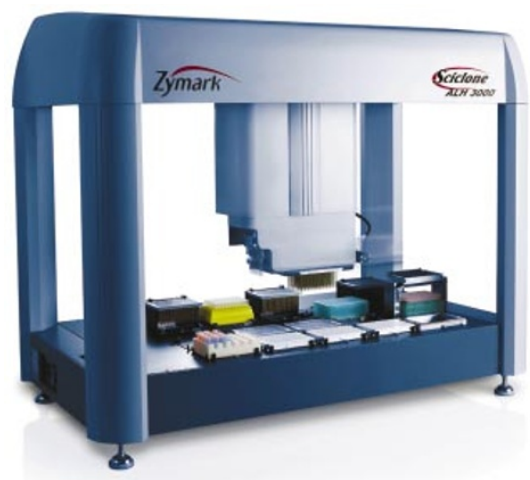

\section{Zymark's latest Sciclone robot brings} added flexibility to liquid handling.

for microplate processing, launched last year by Carl Zeiss of Jena, Germany, incorporates the first commercially available multimode detection system to deliver highperformance data from microplates of up to 1,536 wells. The plate::vision detector reads 96 wells in parallel with three detection modes - absorbance, fluorescence and luminescence - allowing it to analyse a 1,536-well plate in 20 seconds.

\section{All together now}

In theory, high-throughput systems should be able to screen about 100,000 compounds a day against a single disease target. But such rates can rarely be maintained consistently because of problems and delays in transferring sample plates between individual tests and preparation processes. Drug companies typically screen about 25,000 compounds a week, says Mark Beggs, head of consulting at TAP. "What that's really telling you is that coordination of subordinate processes is less than optimal.'

Integrating the various stages of automation into a laboratory can mean that researchers have to adapt to a more industrial production-line environment. "The hard changes are organizational ones, and getting people away from a researchdriven culture to a production-driven culture," says Beggs. That requires attention to bottlenecks and the time taken to transfer plates between individual stages rather than just buying a faster robot, he says. "While some companies get faster by buying the latest thing from the box, more are increasingly becoming faster through process-time reduction."

In addition, commercial highthroughput screening demands extreme flexibility and robustness - a typical screening department in a major drug company will run 20-50 new assays a year against a library of up to 1 million compounds, with each assay involving up to 100 separate activities. Downtime for reconfiguration or replacement of an automated system can significantly reduce effective throughput. Research by automated solutions provider RTS Life Sciences of Manchester, UK, suggests that

\title{
SAMPLED DELIGHTS
}

One of the remaining challenges for high-throughput automation comes at the beginning of many processes - the storage and retrieval of samples. Keeping track of samples can be a major headache for many labs, with researchers often spending hours locating a single specimen.

A pioneer in this field is Biophile, based in Charlottesville, Virginia. Biophile has developed a fully automated storage and retrieval system, which operates at temperatures down to the $-80^{\circ} \mathrm{C}$ required for storing materials such as RNA and tissue samples. The unit has built-in robotics that pick from a rotating vertical stack of almost 1,000 microplates or racks of cryogenic vials.

This month, the company is launching its individual vial retriever, which can automatically re-rack, scan, weigh and sort vials. The retriever will have a capacity of over 22,000 cryovials, which it can select and deliver at an average rate of 200 per hour. "This could double the productivity and rate of working with samples," says Sean Graves, the company's vice-president for technology.

The storage system is designed to be compatible with a wide range of equipment, and the vial retriever is designed to work with LIMS and to take samples directly to workstations by a conveyor system. As with LIMS, the demand for automated storage systems will be increased by regulatory pressure for drug companies and research labs to maintain the security of medical specimens for long periods of time.

"Automated storage and retrieval systems is the clear application that's going to surprise everyone," says Robin Felder, president of the Association for Laboratory Automation. "It's going to surprise everyone because you're going to have to have this."

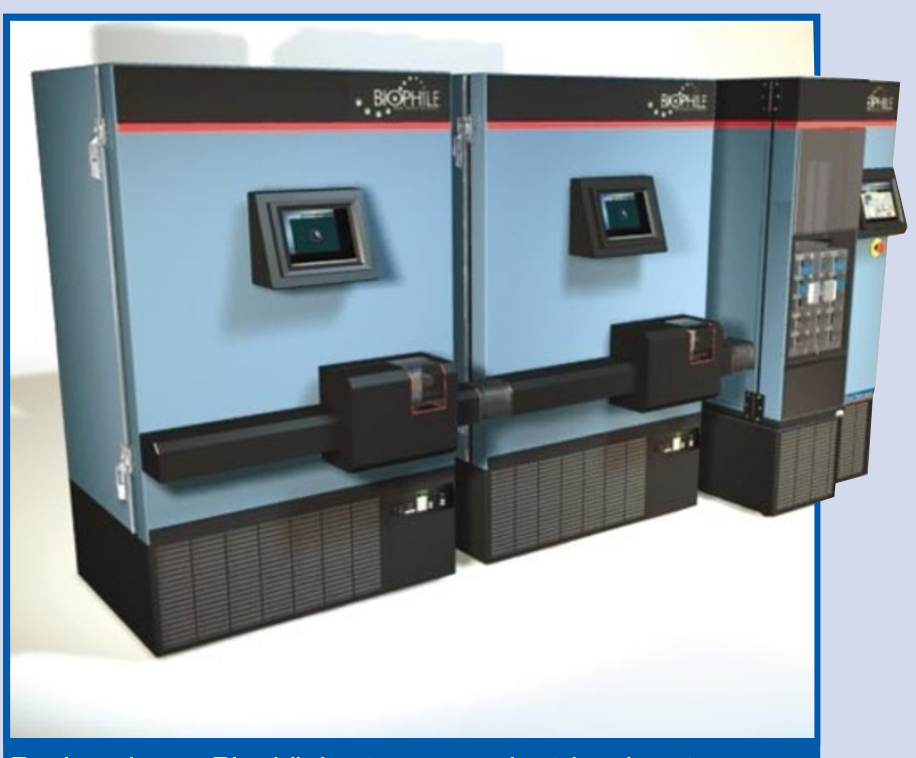

Boxing clever: Biophile's storage and retrieval system. 